\title{
ALGUNOS DATOS SOBRE LA VISIÓN DE LOS NIÑOS Y DE LAS NIÑAS SOBRE LAS CIENCIAS Y DEL TRABAJO CIENTÍFICO
}

\section{SOME DATA ON THE VISION OF CHILDREN ON SCIENCE AND SCIENTIFIC WORK}

\author{
Antonio Pérez Manzano. Universidad de Murcia - España \\ aperez@um.es \\ Antonio de Pro Bueno. Universidad de Murcia - España \\ nono@um.es
}

\begin{abstract}
Resumen Uno de los mayores problemas en el ámbito de la igualdad y su contribución al desarrollo social actual, viene del contraste entre la relevancia para el avance y el crecimiento socioeconómico de las profesiones de elevada cualificación científico tecnológica frente a la acusada escasa presencia femenina en estos estudios. El presente trabajo explora la evolución de Primaria a Secundaria de las actitudes y percepciones procientíficas entre niños y niñas. Se han explorado los conocimientos sobre lo que es Ciencia y sus aportaciones, la percepción global de las ciencias, los científicos y su trabajo y, por último, la valoración de los efectos positivos vs negativos de la ciencia. Los chicos parecen tener un conocimiento algo más adecuado de lo que son productos de las Ciencias, valoran más positivamente sus aportaciones. Las chicas muestran una tendencia a dudar de la utilidad y el efecto de las ciencias sobre el futuro de la sociedad. Los chicos tienden a considerar la profesión científica como más propia de hombres. Las chicas consideran importante el entusiasmo para ser científico, sin embargo, ellas declaran sentirse poco entusiasmadas por la ciencia.
\end{abstract}

Palabras clave Vocación científica, género, percepción de la ciencia, educación científica, percepción de los científicos, actitudes hacia la ciencia.

\begin{abstract}
One of the main problems in the field of equality and its contribution to current social development comes from the contrast between the relevance for the advancement and the socioeconomic growth of the professions of high scientific and technological qualification versus the low female presence in these studies. The present work explores the evolution from Primary to Secondary of attitudes and pro-scientific perceptions between boys and girls. We have explored knowledge about science and its contributions, the global perception of sciences, scientists and their work and, finally, the assessment of the positive and negative effects of science. The boys seem to have a more adequate knowledge of what they are products of the Sciences, they value more positively their contributions. The girls show a tendency to doubt the utility and effect of the sciences on the future of society. Boys tend to consider the scientific profession as men's profession. The girls consider important the enthusiasm to be a scientist, however, they report feeling little excited by science.
\end{abstract}

Keywords Scientific vocation, gender, perception of science, scientific education, perception of scientists, attitudes toward science 


\section{Introducción}

Resultaría difícil encontrar personas que no reconocieran la importancia de las Ciencias en la sociedad actual. El conocimiento científico incide sobre la salud, la conservación ambiental, el bienestar personal y social, la calidad de vida, el ocio y el tiempo libre, la organización social, etc. No es algo ajeno al ser humano y, por ello, son reconocidas como pilares esenciales de lo que somos y de lo que queremos ser (FECYT, 2004, 2006 y 2009). Pero, ¿quiénes son responsables de la formación, de la predisposición hacia ellas o de aprovechar sus valores para la ciudadanía?

Muchos son los "progenitores" de la ciencia que conocen los ciudadanos. Uno de ellos es, sin duda, la "ciencia escolar", esos conocimientos que el profesorado comparte con su alumnado o, por lo menos, que utiliza para instruirlos (Fernández, Gil, Carrascosa y Cachapuz, 2002; Pro, 2002; Pérez y Pro, 2013; Vázquez y Manasero, 2015). No es una ciencia neutra. Es una ciencia intencionada: para preparar para el siguiente nivel educativo, para explicar lo que les gusta a las editoriales... o para atender las necesidades de un ciudadano. Si queremos que los estudiantes aprendan algo porque "lo dice el currículum" trabajaremos unos contenidos. Si queremos que abordemos temas y problemas que le preocupan, tendremos que escuchar antes de seleccionar. Si queremos que el alumnado consuma ciencias estaremos en un modelo. Si queremos que produzca ciencias estaremos en otro. Si queremos que memorice y reproduzca los contenidos que les enseñamos, debemos actuar de una manera. Si queremos que contribuya a hacer un ciudadano que reflexione críticamente o tome decisiones libres ante los problemas sociales, tendremos que utilizar otras estrategias didácticas...

Pero, como dijimos (Pro, 2012) hay otras ciencias en la vida de los estudiantes que posiblemente dejen una huella más profunda o menos reconocible: la "ciencia de la prensa", la "ciencia de la publicidad y del consumo", la "ciencia de los comics y de los dibujos animados", "la ciencia de las series de televisión o de las películas de cine", etc. El ciudadano $-\mathrm{y}$ obviamente los estudiantes lo son- reciben constantemente informaciones, mensajes, adoctrinamientos, gestos persuasivos, y viven experiencias que, de una u otra manera, interiorizan y producen conocimientos, creencias, concepciones, hábitos, actitudes... Todos ellos no sólo condicionan la forma de ser, de pensar, de sentir o de hacer en el ámbito científico escolar, sino que su repercusión se traslada a otras muchas facetas del ser humano.

Por supuesto, también es un progenitor el contexto social y político. Los descubrimientos científicos se han utilizado socialmente de muchas maneras. Así, por ejemplo, mientras unos nos han aportado vacunas, medicamentos, aparatos e instrumentos médicos, fecundación in vitro, etc., otros han contribuido al desarrollo de la industria militar, a la guerra bacteriológica o química, a la carrera armamentista, etc. Mientras unos han mejorado nuestro bienestar (aparatos de música, películas de cine, la radio, móviles...), otros se han utilizado para la propagación de la violencia, del bulling, de valores poco deseables, etc. Mientras unos han posibilitado avances 
positivos para la sociedad (centrales de energía renovables, investigación contra el cáncer, materiales que mejoran la seguridad, etc.), otros han contribuido a la aparición de fenómenos peligrosos (contaminación, cambio climático, alteraciones en ecosistemas, etc.). Mientras unos han comprado los productos de la ciencia (el AVE, internet, videoconsolas...), otros no han podido (desertización, escasez de agua, hambre...).

Por último, hay algo que muchas veces se ignora: lo que hoy un ciudadano necesita saber, saber hacer o saber hacer con otros no será igual a lo que se necesite en 10015 años. Por ello, un elemento clave de la educación formal -y probablemente también de la no formal- es que nuestros estudiantes aprendan a aprender. Debemos asumir que sólo una minoría serán científicos y que, sin embargo, todos estarán en contacto con la ciencia: deberán elegir entre "desaladora si" o "desaladora no", "entre nucleares sí o "nucleares no", entre este aparato con unas características y este otro con otras, entre tomar un producto (con una composición y unos efectos) o no hacerlo, etc. La presencia de las Ciencias en blogs, webs divulgativas, foros de noticias o revistas está ahí, delante de los ciudadanos y, nos guste o no, no podemos ignorarlo.

A lo largo de estos últimos años ha ido creciendo el interés por la visión que tiene la ciudadanía de la ciencia, muchas veces conformadas en las primeras etapas vitales; por su relevancia dentro y fuera de la escuela; por su vinculación a contextos llamados "informales" en el entorno más inmediato del individuo... (European Comision, 2005) Algunos autores defienden que existen niveles bajos de confianza en la ciencia respecto a temáticas como la salud, el medio ambiente, etc. (Acevedo, 2005; Vázquez y Manasero, 2008 y 2009; Gil, 2012), lo que nos lleva a situaciones preocupantes cuando "una persona de la calle" debe enfrentarse a las consecuencias positivas y negativas de la ciencia y la tecnología, en su vida cotidiana.

Ahora bien, estas percepciones o visiones sobre las Ciencias -en definitiva, las actitudes- no son innatas. Hay muchos factores, dentro y fuera de la escuela, que están detrás de las que posee o manifiesta una persona adulta hacia los temas de ciencia y tecnología: lo que podríamos llamar la construcción del adulto "científico". Sin duda, es preciso conocer cómo se va construyendo esa forma de ser, pensar o actuar ante la vida, no sólo como un diagnóstico más, sino como un punto de partida que tenga una mirada clara de dónde está y hacia dónde va.

\section{Las actitudes hacia las ciencias}

Se ha tratado de definir el término actitudes y la forma de medirlas (Kind, Jones y Barmby, 2007). Según Rodríguez y Seoane (1989), encontramos tres elementos básicos presentes en la mayoría:

- Un conjunto organizado de convicciones y creencias.

- Una predisposición favorable o desfavorable asociada a este conjunto. 
- Una actuación respecto a un objeto social consecuencia de los dos puntos anteriores.

Podríamos añadirle una cierta estabilidad de ese sistema de convicciones y creencias, pero no vamos a discutir sobre esta característica, al no ser objeto de nuestro trabajo. Lo que sí compartimos es que no sólo es un proceso afectivo, sino que tiene factores cognitivos, experienciales y conductuales. Ahora bien, de esa diversidad encontramos varias acepciones del término actitudes:

- Actitudes hacia los descubrimientos y el trabajo de los científicos

- Actitudes científicas en la realización de una tarea

- Actitudes hacia las materias curriculares de Ciencia

- Actitudes hacia la salud, la conservación del medio, la paz...

- Actitudes hacia la naturaleza de las Ciencias

En un trabajo anterior (Pérez, 2013) hicimos una amplia revisión de las aportaciones realizadas en la investigación en este ámbito. Por lo que pudimos apreciar, son muchas las variables estudiadas: la influencia del nivel educativo, de la disciplina científica, del currículum oficial, de la Comunidad Autónoma, del grado de desarrollo del país, de las propuestas metodológicas que se usan en la enseñanza, etc.

Entre las variables estudiadas una de las que preocupan es la dependencia del género, probablemente para tratar de explicar el volumen desigual de vocaciones en las carreras científico-tecnológicas. En los resultados hemos podido apreciar que a veces se han impuesto estereotipos, tópicos y tradiciones sociales a la hora de vincular determinadas profesiones a esta variable (Murphy y Beggs, 2003; Osborne, Simon y Collins, 2003; Sjøberg y Schreiner, 2005; Jenkins y Pell, 2006; Vázquez y Manassero, 2007a, 2007b, 2008, 2009; Marbá y Márquez, 2010, etc.). Según la mayor parte de los autores inicialmente presuponen que los resultados deseables se dan más en los chicos que en las chicas. Creemos que era necesario realizar un estudio -con una muestra amplia- para tener más datos sobre la situación actual en nuestro contexto, no sólo para conocer mejor la realidad sino para poder articular propuestas y acciones que incidieran en las situaciones problemáticas. En este contexto, nos hemos planteado: ¿Hay dependencia del género en la visión de las Ciencias y del trabajo de los científicos entre niños y adolescentes en nuestro sistema educativo?

\section{Diseño de la Investigación}

\subsection{Selección y descripción de la muestra}

En un trabajo de carácter demoscópico es preciso definir la población. Ésta se basó en los datos oficiales del Ministerio de Educación, Cultura y Deporte. Se eligieron los últimos cursos de cada etapa de la educación obligatoria -6º de Educación Primaria (EP) y $4^{\circ}$ de Educación Secundaria Obligatoria (ESO)- ya que eran los niveles más adecuados para nuestros propósitos.

Por otro lado, se fijaron unos criterios muestrales. En nuestro caso, se usó un 
muestreo por conglomerado y bietápico. Los estratos utilizados fueron el nivel educativo (ya mencionado), las Comunidades Autonómicas y el tipo de centros (públicos y privados). En cuanto a las unidades de selección, las primarias fueron los centros -tamaño y localización provincial y comarcal- y las secundarias fueron las aulas (en función de la disposición a colaborar). Una descripción más detallada se encuentra en Pérez y Pro (2005) y Pérez (2013). En la Tabla 1 se recogen algunas características de la muestra.

Tabla 1. Algunos datos que describen la muestra

\begin{tabular}{|c|c|c|c|c|}
\cline { 2 - 5 } \multicolumn{1}{c|}{} & \multicolumn{2}{|c|}{$\begin{array}{c}\text { Educación Primaria } \\
\text { No de aulas } \\
\text { alumnos de }\end{array}$} & \multicolumn{2}{c|}{$\begin{array}{c}\text { Educación Secundaria } \\
\text { No de aulas } \\
\text { alumnos }\end{array}$} \\
\hline $\begin{array}{c}\text { Centros } \\
\text { públicos }\end{array}$ & 165 & 2591 & 124 & 1922 \\
\hline $\begin{array}{c}\text { Centros } \\
\text { privados }\end{array}$ & 80 & 1304 & 59 & 1010 \\
\hline $\begin{array}{c}\text { Total de la } \\
\text { muestra }\end{array}$ & 245 & 3895 & 183 & 2932 \\
\hline
\end{tabular}

Fuente: (Pro y Pérez, 2014)

Finalmente se ha trabajado con 6827 cuestionarios (3475 chicos, 3308 chicas y 44 que no indicaron el género). El error muestral por nivel educativo es del $0.8 \%$ en EP y del $0.9 \%$ en ESO y, para el conjunto de la muestra, es del $0.01 \%$.

\subsection{Instrumento de recogida de información}

Hemos utilizado el cuestionario PANA (http://www.um.es/pana/pana/Inicio.html), diseñado específicamente para esta investigación. En la Tabla 2 se recogen algunas de las dimensiones estudiadas, los ítems que las configuraban y los objetivos de cada uno de ellos.

Tabla 2. Cuestionario PANA

\begin{tabular}{|l|l|l|}
\hline \multicolumn{1}{|c|}{ Dimensión } & \multicolumn{2}{|c|}{ Objetivos } \\
\hline $\begin{array}{l}\text { Ciencia y sus } \\
\text { descubrimientos }\end{array}$ & $\begin{array}{l}\text { Ítem 1 } \\
\text { Ítem 2 }\end{array}$ & $\begin{array}{l}\text { Identificar objetos que se deban a descubrimientos de la Ciencia. } \\
\text { Identificar aportaciones realizadas por la Ciencia en la vida } \\
\text { cotidiana. } \\
\text { Identificar cosas que no se podrían hacer sin las aportaciones de } \\
\text { las Ciencias. }\end{array}$ \\
\hline $\begin{array}{l}\text { Visión de la } \\
\begin{array}{l}\text { Ciencia y de los } \\
\text { científicos }\end{array}\end{array}$ & $\begin{array}{l}\text { Ítem } 4 \\
\text { Ítem } 5\end{array}$ & $\begin{array}{l}\text { Dar la visión de la Ciencia y sus aportaciones cotidianas. } \\
\text { Dar la visión de los científicos y sus aportaciones cotidianas. }\end{array}$ \\
\hline
\end{tabular}




\begin{tabular}{|l|l|l|}
\hline \multicolumn{1}{|c|}{ Dimensión } & \multicolumn{2}{|c|}{ Objetivos } \\
\hline $\begin{array}{l}\text { Visión dicotómica } \\
\text { de las Ciencias }\end{array}$ & Ítem 6 & $\begin{array}{l}\text { Posicionarse ante planteamientos dicotómicos de beneficios y } \\
\text { perjuicios (respecto a las contribuciones para la salud vs. industria } \\
\text { militar; mejorar el bienestar vs. aislamiento personal; avances } \\
\text { para mejorar el medio ambiente vs. contaminación; noticias } \\
\text { contradictorias; avances vs. diferencias sociales; y valoración } \\
\text { global). }\end{array}$ \\
\hline $\begin{array}{l}\text { Aprendizaje } \\
\text { formal de las } \\
\text { Ciencias }\end{array}$ & Ítem 7 & $\begin{array}{l}\text { Valorar la influencia de las asignaturas de Ciencias en la vida } \\
\text { cotidiana y el grado de satisfacción de las mismas. } \\
\text { Elegir las temáticas científicas que más interesan a los } \\
\text { encuestados. } \\
\text { Valorar las actividades que se realizan en las clases de Ciencias. }\end{array}$ \\
\hline $\begin{array}{l}\text { Aprendizaje no- } \\
\text { formal de las } \\
\text { Ciencias }\end{array}$ & Ítem 9 & $\begin{array}{l}\text { Ítentificar temáticas sobre los que se charla en el ámbito familiar } \\
\text { ycon los amigos. } \\
\text { Identificar si se visitan museos de carácter científicos. } \\
\text { Identificar programas, documentales, dibujos animados, etc. } \\
\text { relacionados con las Ciencias. } \\
\text { Identificar juegos, juguetes, videojuegos, páginas de internet, etc. } \\
\text { relacionados con las Ciencias. } \\
\text { Identificar libros y revistas relacionados con las Ciencias. }\end{array}$ \\
\hline $\begin{array}{l}\text { Valoración social } \\
\text { de la profesión de } \\
\text { científico y de la } \\
\text { investigación } \\
\text { científica }\end{array}$ & Ítem 13 Ítem 16 16 & $\begin{array}{l}\text { Valorar la profesión de científico en la vida cotidiana. } \\
\text { Valorar las preferencias profesionales de los estudiantes. } \\
\text { Distribuir un presupuesto entre diferentes proyectos o ámbitos de } \\
\text { la sociedad, en función de las prioridades personales. }\end{array}$ \\
\hline
\end{tabular}

Aunque la cantidad de información recogida ha sido ingente (por el número de participantes y la cantidad de cuestiones planteadas), en este trabajo no podemos profundizar en todos los bloques ni en los aspectos concretos de cada uno, por las limitaciones lógicas de espacio.

El Cuestionario PANA fue pasado en un intervalo de tres meses. Contactamos con unos coordinadores de zona de todo el estado español. Se encargaron de seleccionar los centros (unidades primarias de muestreo) y las aulas (unidades secundarias). Las instrucciones que se les enviaron se recogen en la web del proyecto (http://www.um.es/pana/pana/Inicio.html). Participaron 46 profesores -universitarios, de educación secundaria y maestros- de las diferentes Comunidades Autónomas. Se les facilitó el mismo protocolo de aplicación (presentación del cuestionario, observaciones y explicaciones que no influyeran en las opiniones de los entrevistados). Podemos decir que no hubo incidencias destacables, según los aplicadores.

\section{Resultados: descripción y análisis}

Para describir y analizar los resultados -obligadamente sintetizados- los hemos organizado en función de los bloques o dimensiones del cuestionario. En este trabajo sólo nos centraremos en los tres primeros:

- Conocimiento sobre lo que es Ciencia y lo que son sus aportaciones. 
- Percepción global de las ciencias y sobre los científicos y su trabajo.

- Dicotomías: efectos positivos vs negativos de la ciencia

Los resultados del bloque "Dicotomías: efectos positivos vs negativos de la ciencia" fueron descritos y analizados en Pro y Pérez (2014). No obstante, comentaremos brevemente algunos de los referidos a la variable género.

\subsection{Conocimiento sobre lo que es Ciencia}

Como dijimos, planteamos dos cuestiones para conocer si el alumnado era capaz de identificar logros y productos de la Ciencia. Se presentaron 8 objetos y 12 aportaciones. Al respecto hemos de señalar:

- en general, el porcentaje de éxito es alto tanto en los chicos como en las chicas (más de un $85 \%$ en ambos colectivos obtienen resultados "Medios", "Aceptables" o "Bastante buenos"). Es cierto que planteamos algunas situaciones que, según su interpretación, podían tener diferentes "respuestas deseables" pero, a pesar de esta dificultad añadida, los valores globales ponen de manifiesto que ambos colectivos conocen lo que son los productos de las Ciencias e identifican los que no lo son.

- hay objetos o productos en los que los resultados son favorables a los chicos (por ejemplo, "el pan", "las bombas nucleares", "los coches de Fórmula 1", "la revolución industrial", ...); mientras que las chicas los tienen mejores en otros (por ejemplo, "la moda", "las reglas de fútbol", "una serie de TV", "los horóscopos", ...).

- en la Gráfica 1 se recoge la distribución de los resultados globales en función del género. Globalmente se observan diferencias en favor de los chicos. Aunque en la ESO estas diferencias se reducen, siguen siendo favorables a los chicos.

Gráfica 1. Conocimiento sobre los productos de las Ciencias: valoración global según el género

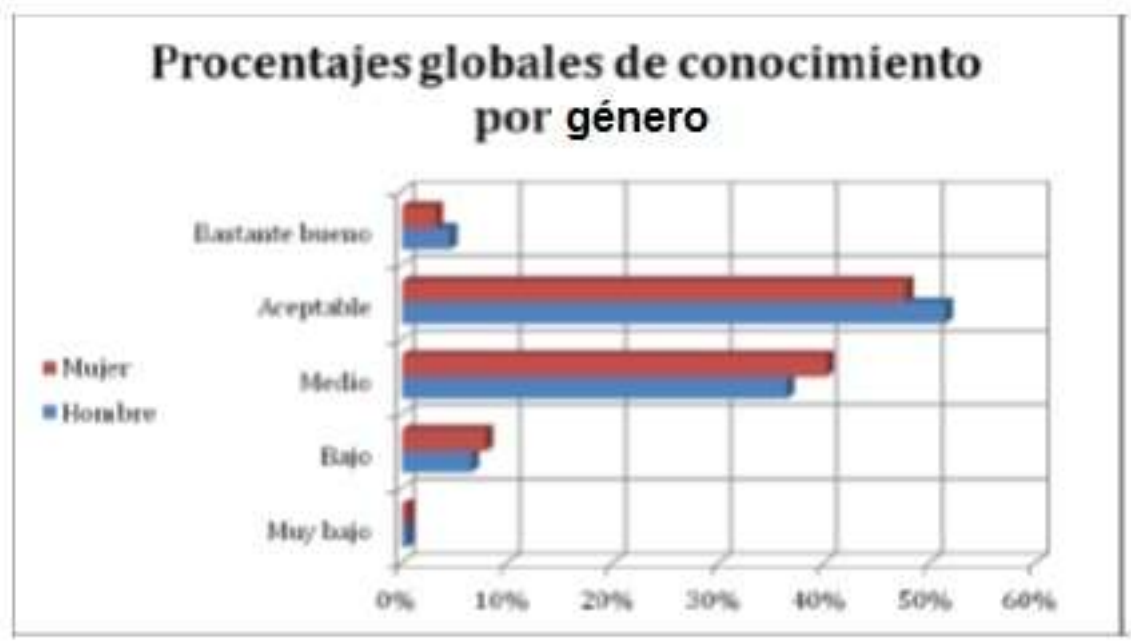


Aunque no son relevantes dichas diferencias, creemos que se debería profundizar en las razones que las justifican. Parece que, en algunas de las cuestiones menos discutibles, los resultados de las chicas son mejores... ¿Qué habrán pensado en los otros casos? Al reducirse con la educación formal, ¿pueden existir factores externos que las hayan propiciado?

\subsection{Percepción de la Ciencia y de los científicos}

En general, la percepción global sobre la ciencia fue positiva (casi un $70 \%$ la consideró "Muy positiva" o "Positiva"). Es cierto que la percepción en EP es más deseable que en la ESO, lo que parece repetirse en otras investigaciones (Pell y Jarvis, 2001; Gibson y Chase, 2002; Murphy y Beggs, 2003; Lindahl, 2005; George, 2006; Barmby, Kind y Jones, 2008; Vázquez y Manassero, 2008; Marbá y Márquez, 2010...). ¿Cuánto más tiempo está un estudiante en la institución escolar la percepción es más negativa? ¿Es una consecuencia de la ciencia que enseñamos? ¿De un aumento de conocimientos o de un tratamiento de las materias científicas que lleva al rechazo o al aburrimiento?

No obstante, centrándonos en el género, como se observa en el Gráfico 2, las percepciones sobre las Ciencias son sensiblemente más positivas en los chicos (las diferencias, entre las positivas y negativas en estos, es de un $65 \%$, mientras que en las chicas es de algo más de 60\%).

\section{Gráfico 2: Percepción sobre la Ciencia: valoración global según género}

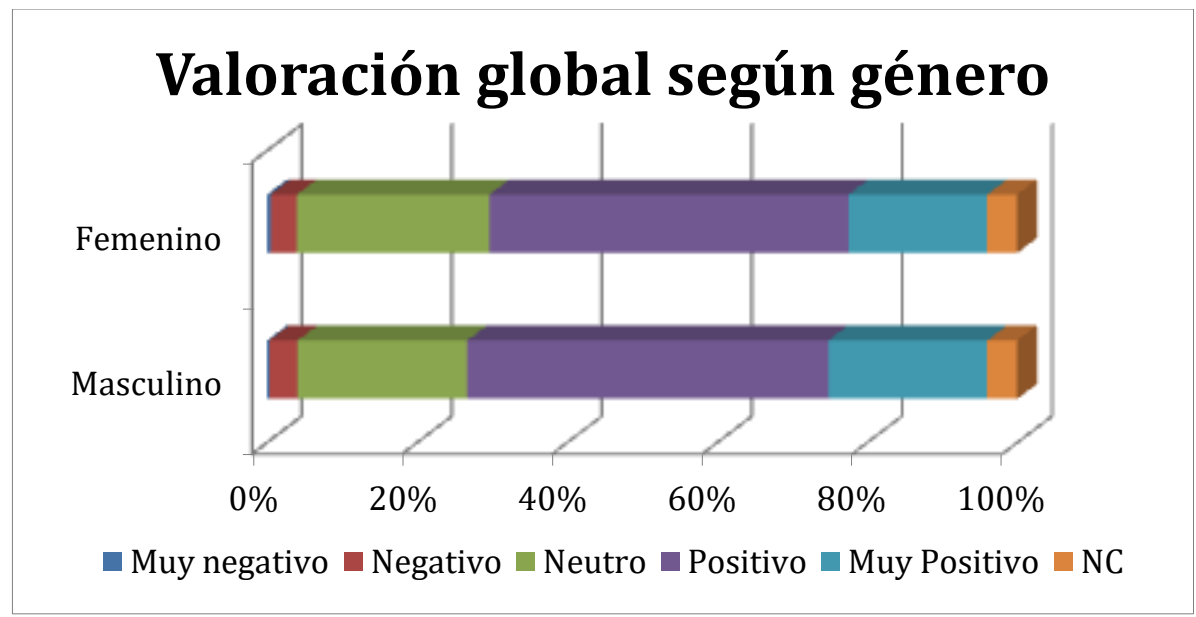

Al respecto hemos de señalar:

- existen diferencias globales sensibles en su acuerdo con "la curiosidad es esencial" y "es tremendamente útil" (en ambas a favor de los chicos) y en su desacuerdo con "deben ser eliminadas de la escuela", "no tienen mucho interés para los no científicos" o "es completamente aburrida" (en éstas el grado de desacuerdo es mayor en las chicas). Como veremos más 
adelante, las chicas defienden más la presencia de las Ciencias en la escuela o en el instituto.

- sin embargo, se observó una tendencia en las chicas a dudar de los efectos de las ciencias sobre el futuro de la sociedad y de la utilidad de las mismas para defendernos de los que quieren engañarnos. Estas diferencias se reducen en la ESO, pero persiste una visión más negativa hacia la Ciencia en las chicas. ¿Son más críticas a la hora de valorar su impacto en nuestra vida cotidiana o simplemente desconocen sus aportaciones $y$ repercusiones sociales?

En cuanto a la percepción de los científicos, en los chicos y en las chicas, el perfil era similar: gana mucho dinero; hace un trabajo divertido; se entusiasma con lo que hace; su trabajo beneficia a todos; no tiene muchos amigos; es capaz de hablar de cosas que no tienen que ver con las ciencias; no sólo trabajan en países ricos; se preocupa por los problemas normales; no trabaja sólo para hacerse famoso; no es despistado; no es una profesión más adecuada a los hombres; está trabajando todo el día; no es una persona divertida; no piensan que sus descubrimientos sean cuestión de suerte; no investigan en lo que quieren; y les discuten sus descubrimientos.

Sin embargo, hay diferencias globales en algunas afirmaciones: "no se preocupa por los problemas normales", "sólo trabaja para hacerse famoso", "es despistado", "es una profesión más adecuada a los hombres", "está trabajando todo el día", "piensan que sus descubrimientos sean cuestión de suerte" y "nadie discute sus descubrimientos"; en todas hay un mayor desacuerdo en las chicas. Es decir, desde nuestra perspectiva, la imagen de las chicas es más ajustada a la realidad.

El considerar la profesión científica como propia de hombres es el bloque que más diferencias genera entre ambos sexos. Es relevante observar como el admitirla como tal es más habitual entre los chicos de Primaria, aunque en ESO se reduce. Las chicas perciben como cualidad relevante en el científico el entusiasmo por lo que hace, creencia que podría estar involucrada o afectar a su toma de decisión vocacional, aunque ya veremos que no es así más adelante.

\subsection{Dicotomías sobre los efectos positivos y negativos de las Ciencias}

Como dijimos, este estudio fue descrito con más meticulosidad en otro trabajo (Pro y Pérez, 2014). Vimos que, cuando deben valorar las contribuciones a la mejora de la salud (vacunas, medicamentos, aparatos médicos...) frente a las aportaciones a la industria militar (bombas, misiles, tanques...), la posición es más positiva hacia los aspectos positivos de las Ciencias. En la misma línea se manifiestan cuando se enfrentan las repercusiones personales (oir música, ver películas, tener juegos...) frente a la comodidad y el individualismo. Y, cuando deben valorar las repercusiones sociales positivas (avances, nuevas profesiones...) y negativas (problemas laborales, 
desequilibrios...) también se posicionan en la visión más favorable hacia las Ciencias.

Sin embargo, ante las noticias de los medios de comunicación, creen que existe un predominio de las negativas hacia las ciencias y admiten que, cuando son contradictorias, muchos suelen dar más credibilidad a las negativas.

Globalmente en la Gráfica 3, se recogen los resultados globales en función del género. Como puede apreciarse, las percepciones son sensiblemente más positivas y menos negativas en los chicos. En este caso, las diferencias entre las positivas y negativas son de un $12 \%$, mientras que en las chicas son de un $9 \%$. También resultan poco significativas.

\section{Gráfica 3. Dicotomías ante las Ciencias: valoración global en función del género}

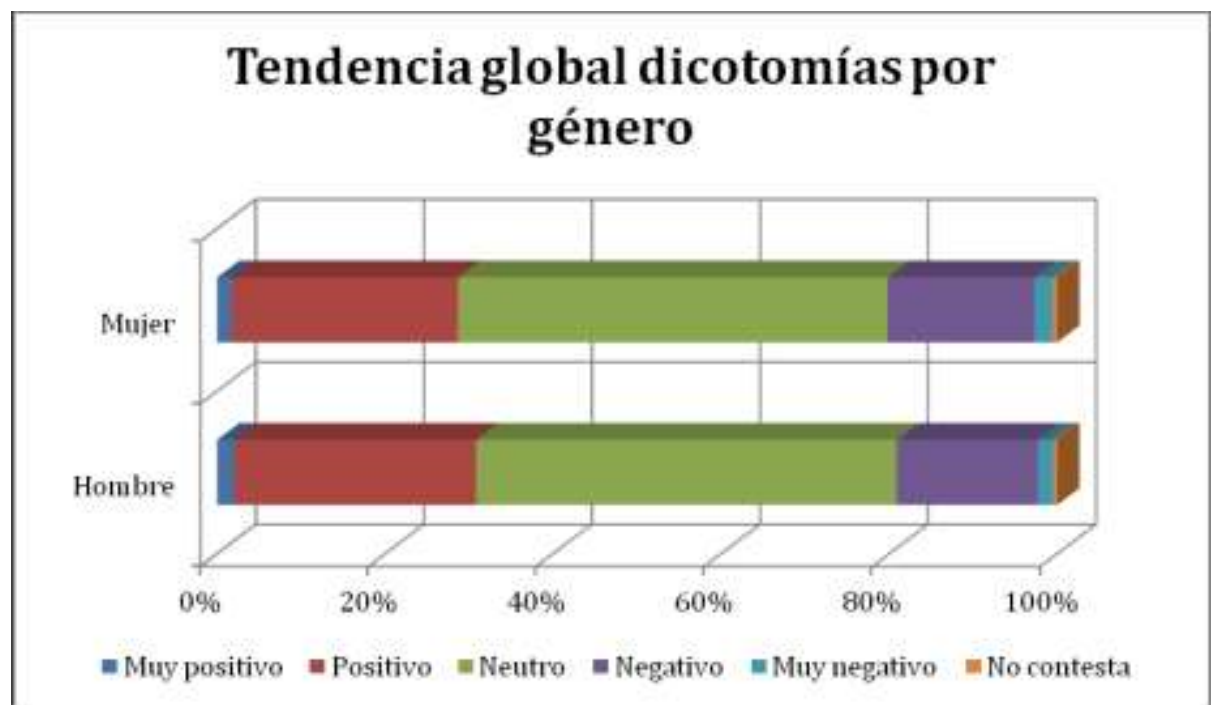

Las opiniones de las chicas son más favorables hacia las ciencias en la dicotomía "salud vs. industria militar"; mientras que los chicos defienden posiciones más favorables la dicotomía "repercusiones personales". En las demás dicotomías existe un equilibrio en las opiniones. Sin embargo, cuando se les plantea globalmente, los chicos son más defensores de las aportaciones de las ciencias (sobre todo en la EP).

Además, las chicas reconocen que hay más noticias negativas, pero coinciden con los chicos en la credibilidad que le dan.

Hay un dato realmente interesante a lo largo de esta dimensión: la presencia mayor de opciones dubitativas ("no lo sé", "opino igual que ambos", "los dos igual", etc.) en las chicas. Nuevamente aparece una visión menos firme y más crítica -aunque las diferencias sean poco relevantes- en este colectivo. Parece que la escolarización en la ESO- reduce estas sensibles diferencias. ¿Es un resultado deseable? ¿Es bueno que no existan dudas... o no? 


\section{Conclusiones}

Nuestra intención era explorar diferentes variables que nos permitieran aportar datos, definir una tendencia, un perfil global de las diferencias en cuanto a la visión de la Ciencia, de sus aportaciones, del trabajo de los científicos, de la incidencia de sus descubrimientos... en función del género. Esta visión es importante conocerla por muchos motivos: necesitamos saber la repercusión de lo que estamos enseñando; si coincide con las necesidades formativas que tiene un ciudadano del siglo XXI; si puede estar directamente asociado a preferencias vocacionales y causante del marcado descenso de la presencia femenina en estudios y profesiones científicas; si existen interferencias sociales que sitúan a nuestros estudiantes ante dicotomías de lo que hay dentro y fuera de los centros escolares; etc.

Hemos podido comprobar que globalmente no existe una dependencia absoluta y marcada en las tres variables-dimensiones estudiadas. No obstante, hemos percibido sensibles y localizadas diferencias que nos han permitido plantearnos cuestiones que deberemos resolver -o, por lo menos, trabajar- en otros trabajos e investigaciones.

Así, detectamos que los chicos parecen tener un conocimiento algo más adecuado de lo que son productos de las Ciencias. Con la escolarización no sólo mejora el porcentaje de aciertos, sino que también se reducen las diferencias en función del género.

Hemos podido apreciar cómo las chicas muestran una tendencia a dudar de la utilidad y el efecto de las ciencias sobre el futuro de la sociedad. No sabemos si considerar un resultado positivo (mayor espíritu crítico) o no tanto (ignorancia del papel de la ciencia en nuestra sociedad). Además, esta tendencia puede resultar relevante en la elección profesional, especialmente si, como hemos comprobado, se acentúa de Primaria a ESO, más próxima a la elección de estudios universitarios.

La exploración del perfil del científico nos ha mostrado una gran homogeneidad en cuanto al género. No obstante, ha aparecido un nuevo escollo: la consideración de la profesión científica como propia de hombres; ha sido la afirmación que más diferencias ha generado entre ambos sexos. Afortunadamente el admitirla como tal es más habitual entre los chicos de Primaria, reduciéndose tras su paso a la ESO.

No obstante, ha habido un dato que nos ha hecho reflexionar. Hemos encontrado que las chicas perciben el entusiasmo como cualidad relevante en el científico por lo que hace. Esta creencia que puede afectar negativamente, por contraste personal, a la elección vocacional: si ellas no "sienten ese entusiasmo, pueden autoexcluirse de esa salida laboral.

En la confrontación entre consecuencias de las ciencias hemos comprobado que, globalmente, los chicos valoran más positivamente las aportaciones de las Ciencias. Sin embargo, existe un mayor equilibrio en las "dicotomías" individualmente; incluso hay algunas en las que las chicas muestran una valoración más positiva, de forma significativa (especialmente en los temas de salud). También reconocen que hay más noticias negativas que positivas, lo que puede interpretarse como una crítica hacia 
este hecho.

Ahora bien, hemos observado una diferencia que consideramos importante: las posiciones de los chicos son más extremas (tanto a favor como en contra); mientras que las de las chicas son más dubitativas, más equilibradas, menos contundentes... Se pueden hacer varias lecturas de la situación: ¿no confían en la ciencia o en los conocimientos científicos?; ¿son más críticas ante las dicotomías planteadas?; ¿aumentarán o disminuirán las dudas con la adquisición de nuevos conocimientos y experiencias?; etc. Y, por último, ¿afectarán estas dudas a la elección de sus estudios profesionales?

El panorama general que nos hemos encontrado en nuestro estudio nos muestra diferentes variables que se deben mejorar si se pretende aumentar la presencia de las chicas en el ámbito científico. Las chicas conocen las ciencias, reconocen su importancia, valoran sus logros..., aunque desconfíen de sus beneficios sociales y no tengan claro con suficiente solidez si realmente suponen una mejora de la calidad de vida en su entorno social próximo. Desde nuestra perspectiva, mostrar una actitud crítica o dubitativa no es un "problema". Todo lo contrario: el uso de la ciencia no es neutral y ser consciente de ello es algo que favorece una visión más adecuada de este ámbito del conocimiento.

Por último, quisiéramos señalar que cualquier programa destinado al fomento de las vocaciones científico-tecnológicas en las chicas debe disponer como elementos esenciales por una parte el cimentar la confianza en los propios conocimientos científicos, aportándoles mayor seguridad y solidez; por otra parte, deben mostrar los beneficios y perjuicios sociales de los avances científicos; y, por último, no se debe olvidar que el entusiasmo es un contenido formativo más de cualquier materia. En cualquier caso, el primer paso, por supuesto sería contar con programas con esta finalidad, algo que hasta ahora resulta inexistente.

\section{Bibliografía}

Acevedo, J.A. (2005). Proyecto ROSE: Relevancia de la Educación Científica. Revista Eureka sobre Enseñanza y Divulgación de las Ciencias, 2 (3), 440-447.

Barmby, P., Kind, P.M. y Jones, K. (2008). Examining changing attitudes in Secondary School Science. Internacional Journal of Science Education, 30(8), 1075-1093.

European Comisión (2004). Europe needs more scientifics. Luxembourg: Office for Oficial Publications of European Communities.

FECYT (2004). Encuesta de Percepción Social de la Ciencia. Madrid.

FECYT (2006). Encuesta de Percepción Social de la Ciencia. Madrid.

FECYT (2009). Encuesta de Percepción Social de la Ciencia. Madrid.

Fernández, I., Gil, D., Carrascosa, J. y Cachapuz, A. (2002). Visiones deformadas de la ciencia transmitidas por la enseñanza. Enseñanza de las Ciencias, 20(3), 477488.

George, R. (2006). A cross-domain análisis of change in students' attitudes toward 
science and attitudes about the utility of science. Internacional Journal of Science Education, 28(6), 571-589.

Gibson, H. L. y Chase C. (2002). Longitudinal impact of an inquiry-based Science Program on Middle School students' attitudes toward Science. Science Education, 86(5), 693-705.

Gil, J. (2012). Actitudes del alumnado español hacia las ciencias en la evaluación PISA 2006. Enseñanza de las Ciencias, 30 (2), pp. 131-152.

Jenkins, E.W. y Pell R.G. (2006). The relevance of Science Education Project (ROSE) in England: a summary of findings. Leeds: University of Leeds.

Kind, P., Jones, K. Y Barmby, P. (2007). Developing attitudes toward science measures. Internacional Journal of Science Education, 29(7), 871-893.

Lindahl, B. (2005). A Longitudinal study about attitudes to science. Comunicación presentada en 4 th ESERA Conference. Barcelona.

Marbá-Tallada, A. y Márquez, C. (2010). ¿Qué opinan los estudiantes de las clases de Ciencias? Un estudio trasversal de sexto de Primaria a Cuarto de la ESO. Enseñanza de las Ciencias, 28(1), 19-30.

Murphy, C. y Beggs, J. (2003). Children perceptions of school science. School Science Review, 84(308), 109-116.

Osborne, J., Simon, S. y Collins, S. (2003). Attitudes toward science: a review of the literature and its implications. Internacional Journal of Science Education, 23(5), 448-467.

Pell, T. y Jarvis, T. (2001). Developing attitude to science scales for use with children of ages from five to eleven years. International Journal of Science Education, 23(8), 847- 862.

Peréz, A. (2013). Actitudes hacia la Ciencia en Primaria y Secundaria. Tesis doctoral. Universidad de Murcia.

Pérez, A. y Pro, A. (2005). Evaluación nacional de actitudes y valores hacia la ciencia en entornos educativos. Madrid: FECYT.

Pérez, A. y Pro, A. (2013). Estudio demoscópico de lo que sienten y piensan los niños y adolescentes sobre la enseñanza formal de las ciencias. En V. Mellado y otros: Las emociones en la enseñanza y el aprendizaje de las Ciencias y de las Matemáticas. Volumen II. (pp. 495-520. Badajoz: Grupo de Investigación DEPROFE.

Pro, A. (2002). ¿Qué procedimientos y actitudes debemos enseñar según los programas de ciencias? Alambique, 33, 37-48.

Pro, A. (2012). Los ciudadanos necesitan conocimientos de ciencias para dar respuestas a los problemas de su contexto. En E. Pedrinaci: 11 ideas clave: El desarrollo de la competencia científica (pp.83-104). Barcelona: Graó.

Pro, A. y Pérez, A. (2014). Actitudes de los alumnos de Primaria y Secundaria ante la visión dicotómica de la Ciencia. Enseñanza de las Ciencias. 32(3), 111-132.

Pro, A.; Pérez, A. y Tárraga, P. (2009). ¿Científico? Sí, pero... Opinión de los escolares 
españoles sobre los científicos y su trabajo. Enseñanza de las Ciencias, Número Extra VIII Congreso Internacional sobre Investigación en Didáctica de las Ciencias, 3649-3656

Rodríguez, A.; Seoane,J. (1989). Creencias, Actitudes y Valores. En J. Mayor y J.L. Pinillos: Tratado de Psicología General (Vol. 7). Madrid: Alhambra.

Sjøberg, S. y Schreiner, C. (2005). Perceptions and images of science and science education. Some results from the ROSE Project in 32 countries. Comunicación presentada en 4 th ESERA Conference. Barcelona.

Vázquez, A. y Manassero, M.A. (2005). La ciencia escolar vista por los estudiantes. Bordón, 57(5), 717-735.

Vázquez, A. y Manassero, M. A. (2007a). En defensa de las actitudes y emociones en la educación científica (I): evidencias y argumentos generales. Revista Eureka sobre Enseñanza y Divulgación de las Ciencias, 4(2), 247-271.

Vázquez, A. y Manassero, M. A. (2007b). En defensa de las actitudes y emociones en la educación científica (II): evidencias empíricas derivadas de la investigación. Revista Eureka sobre Enseñanza y Divulgación de las Ciencias, 4(3), 417-441.

Vázquez, A. y Manassero, M.A. (2008). Las actitudes y la elección de ciencias en la educación obligatoria. Revista Eureka sobre Enseñanza y Divulgación de las Ciencias, 5(3) pp.274-292.

Vázquez, A. y Manassero, M.A. (2009). La relevancia de la educación científica: actitudes y valores de los estudiantes relacionados con la Ciencia y la Tecnología. Enseñanza de las Ciencias, 27(1), 33-48.

\section{Cómo referenciar este artículo/How to reference this article:}

Pérez Manzano, A.; de Pro Bueno, A. (2018). Algunos datos sobre la visión de los niños y de las niñas sobre las ciencias y del trabajo científico. iQUAL. Revista de Género e lgualdad, 1, 18-31, doi: 10.6018/iQual.306091

Pérez Manzano, A.; De Pro Bueno, A.: (2018). Algunos datos sobre la visión de los niños y de las niñas sobre las ciencias y del trabajo científico. [Some data on the vision of children on science and scientific work]. iQUAL. Revista de Género e Igualdad, 1, 18-31, doi: 10.6018/iQual.306091 